\title{
THE STORMERTRON*
}

\author{
WILLARD H. BENNETT \\ U.S. Naval Research Laboratory, Washington, D.C., U.S.A.
}

A tube has been developed in which the shapes of streams of charged particles moving in the earth's magnetic field can be produced accurately to scale. The tube has been named the Störmertron in honor of Carl Störmer who calculated many such orbits. New developments which have made this tube possible include a method for coating the inside of large glass tubes with a transparent electrically conducting film, and an electron gun producing gas-focused streams in less than $\frac{1}{2}$ micron of mercury vapor, a nearly vapor-free grease joint, and a nearly vapor-free carbon black. The magnetic dipole field of the earth is simulated with an Alnico magnet capped with properly shaped soft iron caps. The stream is deflected using two pairs of yoke coils near the gun.

Traversing the stream approximately parallel with the equatorial plane of the earth produces successive shapes assumed by the stream as its direction of projection is advanced past the earth as the stream would be advanced due to the rotation of the sun. Using electronically timed stop motion cameras, motion pictures have been made of the shapes of the advancing streams. It is observed that particles can approach the earth in free orbits and then leave the earth in free orbits if unscattered by collisions, or if properly scattered by collisions while near the earth, the particles become captured into periodic orbits and constrained to move in a ring until scattered out. These rings, as revealed by the Störmertron, consist of particles in periodic orbits which are smeared out in magnetic longitude but are confined to a volume generated by rotating a crescent about the magnetic axis with horns of the crescent impinging on the earth in the two auroral zones.

A larger Störmertron is being built in which the dipole magnetic field at any desired strength can be produced by passing a current through the coil representing the earth. This coil has been designed to produce the magnetic field of the dipole at the center with an error of less than $3 \%$.

* Presented at the Symposium by E. O. Hulburt, Washington. D.C. 
This tube is to be used to plot out various auroral areas in order to compare with the observed geographical distribution of aurorae to be obtained during the International Geophysical Year.

\section{Discussion}

Ferraro: Is it possible to do this experiment with wider beams?

Hulburt: We have not tried that. I think it is difficult to perform it with an electron gun.

Dattner: Was the reason of the discharge inside the forbidden zone the scattering of the electrons by collisions?

Hulburt: Yes.

Block: I wonder if you have calculated the influence of the positive charges which may be produced by ionizing the gas in the vicinity of the beam. This may affect the orbit.

Hulburt: I think in this experiment with this position of the electron gun the electron orbits behave as in field-free space.

Alfvén: We saw very nice ring-currents. Could you say of what type they were?

Hulburt: They are probably due to electrons scattered into ring-shaped curves around the dipole. I think these results would have some bearing on geomagnetic effects such as magnetic storms.

Singer: I would like to point out that the scattering mechanism in these model experiments is different from the scattering mechanism I proposed yesterday. The scattering in the Störmertron is produced by collisions with gas atoms. The scattering process I proposed was due to perturbations of the dipole field several earth radii out. However, the particles that I started with are protons and will of course describe analogous orbits in the dipole field.

Dattner: I would like to ask Dr Hulburt about the mean free path in the chamber.

Hulburt: The pressure is probably about $1 \mathrm{I}^{-4} \mathrm{~mm} \mathrm{Hg}$ and the mean free path some centimeters.

Block: What was the energy of the particles? roo volts or so?

Hulburt: Yes, a few hundred volts.

Block: I believe that the mean free path would be about $\mathrm{I} \mathrm{m}$. 